Probability, Networks and Algorithms

Probability, Networks and Algorithms

PNA Maintenance Routing for Train Units: the Transition Model

G. Maróti, L.G. Kroon

Report PNA-E0415 August 2004 
CWI is the National Research Institute for Mathematics and Computer Science. It is sponsored by the Netherlands Organization for Scientific Research (NWO).

$\mathrm{CWI}$ is a founding member of ERCIM, the European Research Consortium for Informatics and Mathematics.

CWI's research has a theme-oriented structure and is grouped into four clusters. Listed below are the names of the clusters and in parentheses their acronyms.

\section{Probability, Networks and Algorithms (PNA)}

Software Engineering (SEN)

Modelling, Analysis and Simulation (MAS)

Information Systems (INS)

Copyright (C) 2004, Stichting Centrum voor Wiskunde en Informatica

P.O. Box 94079, 1090 GB Amsterdam (NL)

Kruislaan 413, 1098 SJ Amsterdam (NL)

Telephone +31205929333

Telefax +31205924199

ISSN 1386-3711 


\title{
Maintenance Routing for Train Units: the Transition Model
}

\begin{abstract}
Train units need regular preventive maintenance. Given the train units that require maintenance in the forthcoming 1-3 days, the rolling stock schedule must be adjusted so that these urgent units reach the maintenance facility in time. In an earlier paper Maroti and Kroon propose a model that requires a large amount of input data. In this paper we describe a less involved multicommodity flow type model for this maintenance routing problem. We study the complexity of the problem. It turns out that the feasibility problem for a single urgent train unit is polynomially solvable but the optimisation version is NP-hard. Finally, we report our computational experiments on practical instances of NS Reizigers, the main Dutch operator of passenger trains.
\end{abstract}

2000 Mathematics Subject Classification: 90B06; 90B10; 90C35

Keywords and Phrases: Railway transportation; Maintenance routing 


\title{
Maintenance Routing for Train Units: the Transition Model
}

\author{
Gábor Maróti \\ CWI, Amsterdam and \\ NS Reizigers, Utrecht \\ P.O.Box 94079, 1090 GB Amsterdam, The Netherlands \\ E-mail: G.Maroti@cwi.nl \\ Leo Kroon \\ NS Reizigers, Utrecht and \\ Erasmus University, Rotterdam \\ P.O.Box 1738, 3000 DR Rotterdam, The Netherlands \\ E-mail: L.Kroon@fbk.eur.nl
}

\begin{abstract}
Train units need regular preventive maintenance. Given the train units that require maintenance in the forthcoming 1-3 days, the rolling stock schedule must be adjusted so that these urgent units reach the maintenance facility in time. Maróti and Kroon [11] propose a model that requires a large amount of input data. In this paper we describe a less involved multicommodity flow type model for this maintenance routing problem. We study the complexity of the problem. It turns out that the feasibility problem for a single urgent train unit is polynomially solvable but the optimisation version is NP-hard. Finally, we report our computational experiments on practical instances of NS Reizigers, the main Dutch operator of passenger trains.
\end{abstract}

AMS Subject classification: 90B06, 90B10, 90C35

Keywords: Railway transportation, maintenance routing.

\section{Introduction}

The Dutch passenger railway operator NS Reizigers runs a number of train units. The units need regular preventive maintenance checks, say, after every $30,000 \mathrm{~km}$. Units that travelled that distance must go to the maintenance facility. For this, the rolling stock schedule has to be modified so that the 
units, that need maintenance, arrive in time at a maintenance facility while still being deployed for timetable services. As yet NS Reizigers does not use a decision support system for maintenance routing. It is the goal of this research to provide mathematical programming models and solution methods that can serve as basis for such a decision support system.

Maróti and Kroon [11] describe an integer programming model for maintenance routing. Despite promising computational results, the model also has a certain drawback. The issue is that it requires many details about the shunting possibilities; details that are quite difficult to obtain in practice. Therefore we present in this paper a model that requires less input data. Although it is a bit further from reality, we believe that it is detailed enough to produce useful results.

We describe the maintenance routing problem in Section 2. Our new integer programming model is presented in Section 3. In Section 4 we prove some complexity results. Our computational results are in Section 5 .

\section{The maintenance routing problem}

\subsection{Maintenance planning in practice}

The Logistics Department of NS Reizigers determines the rolling stock schedule. We call this the regular plan. It contains the rolling stock movements between different stations but does not involve movements inside stations. These are described by shunting plans created by local shunting crew. A smallest indivisible movement in the regular plan to be carried out by a single train unit is called a task. So, a movement carried out by a train with more than one unit corresponds to several tasks. Two tasks form a regular transition if a train unit carries them out consecutively in the regular plan.

Some of the tasks in the regular plan are maintenance tasks. If a train unit carries out such a task, it undergoes a maintenance check. (Maintenance checks can only take place at specialised stations.) The regular plan assigns maintenance tasks arbitrarily, irrespective of which unit needs to be maintained and when. The actual maintenance routing is handled shortly before execution. NS Reizigers uses a fixed planning horizon for maintenance routing, usually 3 days. Input is the regular plan and a list of urgent train units: those that need a maintenance check within the planning horizon. They may have different urgencies, expressed by a deadline (for instance saying that the unit should 
be maintained within 2 days). The part of the regular plan that lies within the planning horizon must be adjusted so that each urgent unit is routed to a maintenance facility while carrying out timetable services. This maintenance planning process is carried out daily, with a rolling horizon.

The maintenance planners work as follows. They consider a (small) number of train units that are at some moment at the same station and replace their regular transitions by new transitions. The purpose of these changes is to lead the urgent units in time to a maintenance task. Figure 1 shows a small example. Thick lines represent tasks, dashed lines regular transitions, and arrows new transitions. The new duty of the urgent unit contains a maintenance task.

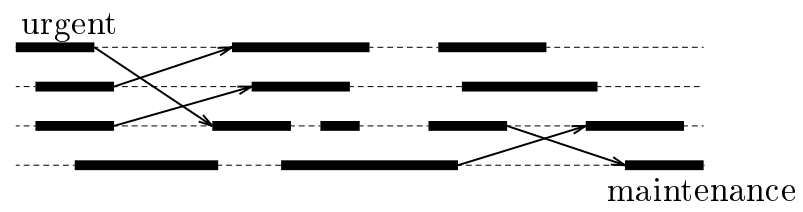

Figure 1

Changes may require shunting at the stations. The maintenance planners do not have direct access to shunting options. Therefore they check with the local shunting crew if the preferred changes can be implemented in practice. If not, maintenance planners must come up with other changes and check them again. Communication between the maintenance planners and the local shunting crew takes time, so the maintenance routing planners cannot test too many possibilities. Therefore they are usually satisfied with the first acceptable solution found.

\subsection{Previous work}

Several related papers can be found in the literature. Lingaya et al. [10] describe a model for operational management of locomotive-hauled railway cars. They seek for a maximum expected profit schedule that satisfies various constraints, among them also maintenance requirements.

Some other papers focus on aircraft maintenance routing. For example, Barnhart et al. [2], Clarke et al. [5], Feo and Bard [8], Gopalan and Talluri [9] and Talluri [13] deal with this problem. Anderegg et al. [1] describe models for railway applications that are similar to the aircraft routing models. 
These models cannot be applied directly to the maintenance routing at NS Reizigers. The models consider maintenance routing as a part of the mediumand long-term vehicle scheduling problem, and specify the tasks of the individual vehicles during the entire planning period. The models above create the vehicle schedule from scratch, without taking shunting issues into account. Therefore in our railway application there is no guarantee that the output of these models can be carried out in practice.

Furthermore, disturbances and delays make it unlikely that the rolling stock schedule can be carried out exactly as planned in a period longer than a couple of days. On the other hand, the Dutch railway network contains a large number of frequently operated relatively short train lines. This provides many exchanging possibilities for the train units, therefore a couple of days is mostly enough to route an urgent train unit to a maintenance facility. This explains why the maintenance planners consider planning periods of length $1-3$ days, and only a small number of train units to be routed.

\subsection{Our approach}

The bottleneck in maintenance routing is shunting: planners should make sure that the desired new transitions can in fact be carried out. A decision support system for maintenance routing should be able to judge the difficulty of the considered solutions. However, generating shunting plans is difficult, even for just a single middle-sized station. Thus we cannot instantly check if it is possible to carry out the shunting operations involved with a change. What we can do is estimate shunting costs.

We know that the regular plan allows a shunting plan. Therefore if we apply only a small number of changes, the modified plan will be close to the regular plan and we may hope that the local shunting crew will be able to carry out the new plan.

Maróti and Kroon [11] propose the "Scenario Model" for the maintenance routing problem. It is based on scenarios; a scenario is a group of transitions for which the whole shunting process can be carried out. The Scenario Model requires many details on shunting, more than is likely to be available in practice. Therefore we describe here another model, the "Transition Model". It is a variant of the multi-depot vehicle routing problem, studied in detail e.g. in Desrosiers et al. [7] and Toth and Vigo [14]. Our results and methods are related to those in Carraresi and Gallo [4] and in Bertossi et al. [3]. 
The Transition Model incorporates no other information on shunting possibilities than a description of which new transitions are allowed; the sole criterion for that is whether there is enough time to carry out the shunting operations involved with the particular transition without causing delays. We then assign a cost value to every allowed new transition and define the cost of a solution as a simple function of the costs of the new transitions in the solution. As the Transition Model is less involved than the Scenario Model, it may produce less acceptable solutions. However, its input data are a lot easier to obtain and in the setting of an interactive decision support system the cost function can be tuned based on rejections or approvals of local shunting crew. Recall that whatever method for maintenance planning is used, each solution needs approval of the shunting crew before it can be implemented anyway.

\section{The Transition Model}

\subsection{The input: maintenance routing graphs}

We formalise the input for our model. A timetable is a set $V$ of tasks together with four integer valued functions Dep_station(), Arr_station(), Dep_time() and Arr_time() on $V$ such that $\operatorname{Dep\_ time~}(v) \leqslant \operatorname{Arr} \_t i m e(v)$ for each $v \in V$. A regular

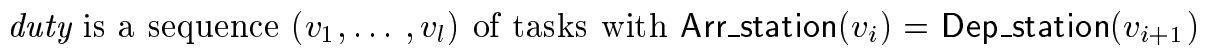
and Arr_time $\left(v_{i}\right)<\operatorname{Dep}$ _time $\left(v_{i+1}\right)$ for each $i=1, \ldots, l-1$. A regular plan is a partition of $V$ into regular duties. Each pair of consequent tasks in such a regular plan is a regular transition. In a railway application, the regular duties are the sequences of tasks assigned to a particular train unit. A candidate transition is a pair of tasks $(v, w)$ such that it is not a regular transition, and such that $\operatorname{Arr} \_s t a t i o n(v)=\operatorname{Dep\_ station}(w)$ and $\operatorname{Dep} \_t i m e(w) \geqslant \operatorname{Arr} \_t i m e(v)+$ Buffer_time where Buffer_time is a given number.

All graphs in this paper are directed. A source in a graph is a node with no in-going arcs and a sink is a node with no out-going arcs. The set of tasks $V$ of a timetable and the set of regular and candidate transitions form an acyclic graph. (A graph is acyclic if it does not contain directed cycles.) Any graph that can be obtained in this way is called a maintenance routing graph (or MR-graph).

An urgency in an MR-graph $G=(V, A)$ is a function $M$ on a subset $U$ of the set of sources in $G$ with $M(u) \subseteq V$ for each $u \in U$; members of $U$ are urgent nodes. In a maintenance routing application a node is urgent if it is the earliest task of one of the train units that must reach a maintenance facility 
in the planning period; for $u \in U, M(u)$ is the set of maintenance tasks that can be assigned to $u$ in order to satisfy the maintenance requirement of the corresponding train unit.

In real world maintenance routing, we may allow the buffer time to vary from arc to arc, but here an MR-graph always has a uniform buffer time. It also happens in real world maintenance routing that some practical instances can only be solved when allowing non-scheduled train movements without passengers. If desired we can model such movements by transitions between tasks that arrive and depart at different stations. However, such more general transitions are not part of our definition of MR-graphs.

\subsection{The model}

From the timetable and Buffer_time we construct the corresponding MR-graph.

The sequences of tasks that can be assigned to a single train unit over a whole planning period are precisely the directed paths in the corresponding MR-graph that connect a source to a sink. A path cover in an acyclic graph $G=(V, A)$ is a set of arcs that forms a collection of node disjoint paths such that the paths cover $V$ and that each path connects a source of $G$ with a sink of $G$; the regular transitions in a maintenance routing graph form a path cover. So, the maintenance routing problem amounts to finding a path cover such that the path in the path cover that starts at urgent node $u$ contains a node in $M(u)$.

In order to evaluate shunting efforts, we calculate for each transition a cost value estimating how difficult it is to use it for a train unit (see Section 5.1 for a short discussion on how we calculate these estimates.) We measure the overall shunting difficulty of a solution by the sum of the individual transition costs.

With these data, the maintenance routing problem becomes the following integer multicommodity flow model; we define it for general acyclic graphs for later reference.

Transition Model: Given an acyclic graph $G=(V, A)$, a set $U$ of sources and an urgency $M$ on $U$, as well as a cost function $c: A \rightarrow \mathbb{R}_{+}$, find a path cover $Z$ minimising

$$
\sum_{a \in Z} c(a)
$$

such that for each $u \in U$, the path starting at $u$ contains a member of $M(u)$. 
We formulate this as a binary linear program. The variables are:

$$
z: A \rightarrow\{0,1\} \text {, and for each } u \in U, x_{u}: A \rightarrow\{0,1\} .
$$

The functions $x_{u}$ are network flows indicating the paths of the urgent units, while $z$ is the characteristic function of a path cover.

The constraints are:

1. flow conservation for $z$ at every node $v$ except for the sources and sinks; each node has throughput 1 in $z$ ( $\delta^{\text {in }}$ and $\delta^{\text {out }}$ denote the set of entering and leaving arcs, respectively):

$$
z\left(\delta^{\text {in }}(v)\right)=z\left(\delta^{\text {out }}(v)\right)=1 ;
$$

2. flow conservation for each flow $x_{u}$ at every node $v$ except if $v=u$ or $v \in M(u)$ :

$$
x_{u}\left(\delta^{\text {in }}(v)\right)=x_{u}\left(\delta^{\text {out }}(v)\right) ;
$$

3. the flows $x_{u}$ must form a subsystem of the flow $z$ :

$$
\text { for every arc } a: \sum_{u \in U} x_{u}(a) \leqslant z(a) ;
$$

4. every node $u \in U$ has out-flow 1 in $x_{u}$; every source $v^{\prime}$ has out-flow 1 in $z:$

$$
x_{u}\left(\delta^{\text {out }}(u)\right)=1 ; \quad z\left(\delta^{\text {out }}\left(v^{\prime}\right)\right)=1 ;
$$

5. a maintenance task $v$ has neither any out-going flow in any of the flows $x_{u}$, nor any in-going flow in a flow it is not allowed for:

$$
\sum_{u \in U} x_{u}\left(\delta^{\mathrm{out}}(v)\right)=0 ; \quad \sum_{u \in U: v \notin M(u)} x_{u}\left(\delta^{\mathrm{in}}(v)\right)=0 .
$$

The objective function is:

$$
\text { minimise } \sum_{a \in A} c(a) \cdot z(a) \text {. }
$$




\section{Reducing the problem size}

An arc in an acyclic graph $G$ is said to be covered if it is in at least one path cover of $G$. Many transitions in a maintenance routing problem will have a very long time span; such transitions will typically not be covered in the corresponding MR-graph, so they make the model larger than necessary. We call an acyclic graph reduced if each arc is covered. Finding a path cover and determining which arcs are covered are network flow problems, so we can reduce a graph in polynomial time. Actually when a path cover is given, as in the case in MRgraphs, one can determine the covered arcs in linear time. In spite of the fact that this is standard network flow theory (for details see e.g. Schrijver [12]), we explain it as we need the construction later. Given an acyclic graph $G=(V, A)$ with a path cover $R$, construct an auxiliary graph $\widehat{G}=(\widehat{V}, \widehat{A})$ as follows: for each $v \in V$ there are two distinct nodes $v_{\text {out }}$ and $v_{\text {in }}$ in $\widehat{V}$. For each candidate transition $v w \in A$, there is an $\operatorname{arc} v_{\text {out }} w_{\text {in }}$ in $\widehat{A}$, and for each regular transition $v w \in A$, there is an $\operatorname{arc} w_{\text {in }} v_{\text {out }}$ in $\widehat{A}$. A small example is given in Figure 2. Now an arc in $G$ is covered if and only if it is either in $R$ or the corresponding arc in $\widehat{G}$ is in a directed circuit. Therefore identifying the covered arcs amounts to finding the strong components of $\widehat{G}$ which can be done in linear time, indeed.

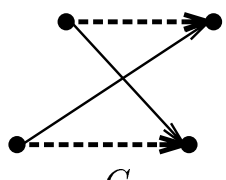

G

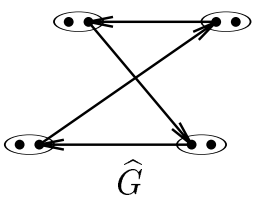

Figure 2: The auxiliary graph. (The dashed arcs are regular.)

\section{Complexity results}

In this section we derive complexity results for the Transition Model. We prove that the feasibility problem is NP-complete even when there is only a single urgent node. When the number of sources is fixed, an optimal solution to the Transition Model can be found in polynomial time with dynamic programming irrespective the number of urgent nodes. For MR-graphs with one urgent node, the feasibility version is polynomially solvable, but the optimization problem remains NP-hard. The following table summarizes these results of this section. 


\begin{tabular}{l|c|c}
\hline Complexity of the Transition Model & Feasibility & Optimising \\
\hline Arbitrary acyclic graphs, $|U|=1$ & NP-hard & NP-hard \\
Arbitrary acyclic graphs, fixed number of sources & $\mathrm{P}$ & $\mathrm{P}$ \\
MR-graphs, $|U|=1$ & $\mathrm{P}$ & NP-hard \\
\hline
\end{tabular}

\subsection{NP-hardness in arbitrary acyclic graphs}

Theorem 1. It is NP-complete to decide if an acyclic graph has a path cover such that one of its paths connects a given source with a given sink.

Proof. We reduce the satisfiability problem to our problem. Let $x_{1}, \ldots, x_{n}$ be Boolean variables, let $\varepsilon \in\{ \pm 1\}$ and denote

$$
x_{j}^{\varepsilon}=\left\{\begin{aligned}
x_{j} & \text { if } \varepsilon=+1, \\
\neg x_{j} & \text { if } \varepsilon=-1 .
\end{aligned}\right.
$$

Consider the disjunctive normal form

$$
\varphi=\bigwedge_{i=1}^{k}\left(x_{i_{1}}^{\varepsilon_{i_{1}}} \vee x_{i_{2}}^{\varepsilon_{i_{2}}} \vee \cdots \vee x_{i_{m(i)}}^{\varepsilon_{i_{m(i)}}}\right)
$$

It is well-known that it is NP-complete to decide whether or not we can assign values to the variables that make $\varphi$ true (Cook [6]). We start with an empty graph. We insert two nodes $a_{j}, b_{j}$ for each variable $x_{j}$ and draw an $\operatorname{arc} a_{j} b_{j}$. For each $i=0, \ldots, k$, we create three nodes $s_{i}, c_{i}, d_{i}$, and insert the $\operatorname{arcs} c_{i} s_{i}$, $s_{i} d_{i}$ and $c_{i} d_{i}$. Moreover, we insert the arc $c_{k} d_{0}$. Let $s=c_{0}$ and $t=d_{k}$.

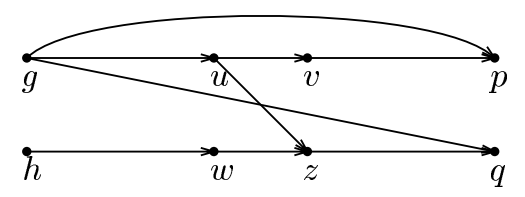

Figure 3

For the occurrence of a variable $x_{j}$ (either as $x_{j}$ or as $\neg x_{j}$ ) in clause $i$, we create a "box" as shown in Figure 3. We create 8 new nodes $u, v, w, z, g, h, p, q$ and draw the arcs $u v, u z, w z, g u, h w, v p, z q, g p$, and $g q$. We insert the arc $s_{i-1} u$. Moreover, we draw the arc $v s_{i}$ if the non-negated $x_{j}$ occurs in clause $i$, and we draw the arc $z s_{i}$ if $\neg x_{j}$ occurs in clause $i$.

We order the occurrences of $x_{j}$ by increasing clause index. We draw an arc from $a_{j}$ to the $v$-node of the first $x_{j}$-box. We insert an arc from the $w$-node of 
any $x_{j}$-box to the $v$-node of the next $x_{j}$-box. Finally, we join the $w$-node of the last $x_{j}$-box to $b_{j}$. Then we obtain a picture as in Figure 4 . Note that the arcs $a_{j} b_{j}, c_{i} s_{i}, s_{i} d_{i}, g u, u v, v p, h w, w z, z q$ form a path cover.

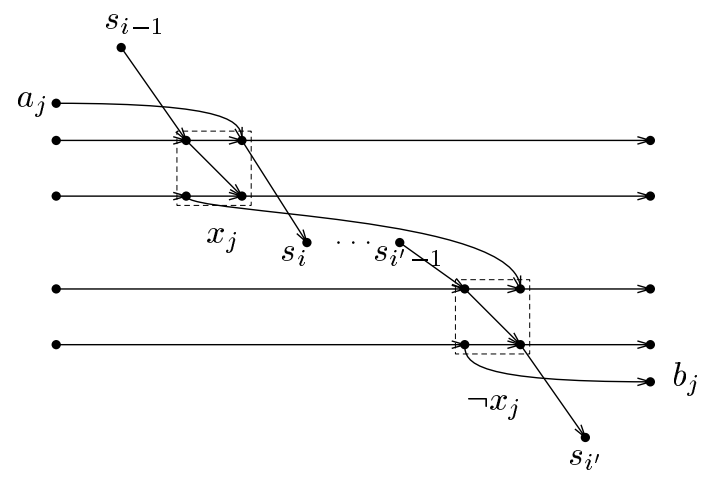

Figure 4

We claim that a path cover connecting $s$ with $t$ exists in this graph if and only if $\varphi$ is satisfiable.

Suppose there exists such a path cover. Observe that for any pair of occurrences of $x_{j}$, the paths use in both boxes either the $u v$-arc or the $u z$-arc. This follows easily for two consecutive occurrences (i.e. for those that are joint by a $w v$-arc) using the fact that the $v$-nodes and the $z$-nodes have exactly two entering arcs while the $u$-nodes and the $w$-nodes have exactly two leaving arcs. Set $x_{j}$ true if the $u v$-arcs are used in the $x_{j}$-boxes, otherwise set it false. Observe that every $s-t$ path in the graph contains all the nodes $s_{i}$. Then the $s-t$-path proves that every clause is satisfied.

Conversely, suppose that the variables have an assignment satisfying $\varphi$. For any clause $i$, label any of the literals that makes it true.

If $x_{j}$ has value true, select the $\operatorname{arc} a_{j} b_{j}$, as well as the $u v, w z, h w$ and $z q$ arcs of all the $x_{j}$-boxes. If an occurrence of $x_{j}$ is labelled for clause $i$, select the $s_{i-1} u, v s_{i}$ and $g p$-arcs of this box, otherwise select the $g u$ and $v p$-arcs.

If $x_{j}$ has value false, select the $u z, h w$ and $v p$-arcs of the $x_{j}$-boxes, the $w v$ arcs between the $x_{j}$-boxes as well as the arcs connecting the nodes $a_{j}$ and $b_{j}$ to the $x_{j}$-boxes. If the occurrence is labelled for clause $i$, select the $\operatorname{arcs} s_{i-1} u, z s_{i}$ and $g q$ in this $x_{j}$-box, otherwise select the arcs $g u$ and $z q$.

Finally, select the $\operatorname{arcs} c_{i} d_{i}$ for $i=1, \ldots, k-1$ and the $\operatorname{arc} c_{k} d_{0}$. Then the 
selected arcs form a path cover connecting $s$ with $t$.

\subsection{Bounded number of train units}

In the maintenance routing application, the number of sources equals the number of train units of the given type. We can consider it as a constant, while the length of the planning period (therefore the number of nodes and arcs in the graph) is the varying parameter. Then the Transition Model can be solved in polynomial time.

Theorem 2. For any acyclic graph $G=(V, A)$, the optimisation problem (1) - (7) can be solved in $O\left((|V||A|)^{O(d)}\right)$ time where $d$ is the number of sources.

Proof. Let $n=|V|, m=|A|$, and let $k=|U|$. We assume that the sources are $S=\{1, \ldots, d\}$ and that $U=\{1, \ldots, k\}$. The optimisation problem can be reduced to a shortest path problem in an acyclic graph $G^{\prime}=\left(V^{\prime}, A^{\prime}\right)$ with at most $(2 m n)^{d}$ nodes and at most $(2 m n)^{2 d}$ arcs. The sketch of the construction is as follows.

We colour the nodes of $G$ red. By subdividing arcs, we can transform $G$ into a graph where the node set is partitioned into disjunct subsets $V_{0}, \ldots, V_{\ell}$ such that each $\operatorname{arc} a$ connects $V_{i}$ to $V_{i+1}$ for some index $i$. We also assume that the arcs between $V_{0}$ and $V_{1}$ form a matching of size $d$. The enlarged graph has at most $m n$ nodes and at most $m n$ arcs. We colour the newly created nodes black. Then a path cover in the original graph corresponds to a system of paths in the enlarged graph covering all the red nodes.

We create a member of $V^{\prime}$ for every $(d+k)$-tuple $v^{\prime}=\left(a_{1}, \ldots, a_{d}, \varepsilon_{1}, \ldots, \varepsilon_{k}\right)$ where $(i)$ the $\operatorname{arcs} a_{i}$ lie between the subsets $V_{t-1}$ and $V_{t}$ for some index $t$, (ii) the $\operatorname{arcs} a_{i}$ cover all the red nodes in $V_{t-1} \cup V_{t}$, and $(i i i) \varepsilon_{j} \in\{0,1\}$ for each $j=1, \ldots, k$. The $\operatorname{arcs} a_{i}$ indicate the routes of the train units through the network, while $\varepsilon_{j}$ encodes whether urgent unit $j$ has been maintained so far.

Let $s^{\prime}$ be the node in $V^{\prime}$ with $\operatorname{arcs} a_{i}$ between $V_{0}$ and $V_{1}$ and with $\varepsilon_{1}=\cdots=$ $\varepsilon_{k}=0$. Let $T^{\prime}$ contain all nodes $v^{\prime} \in V^{\prime}$ such that the $\operatorname{arcs} a_{i}$ lie in $v^{\prime}$ between the subsets $V_{\ell-1}$ and $V_{\ell}$, and such that $\varepsilon_{j}=1$ for each $j$.

Let $v^{\prime}=\left(a_{1}, \ldots, a_{d}, \varepsilon_{1}, \ldots, \varepsilon_{k}\right)$ and $w^{\prime}=\left(b_{1}, \ldots, b_{d}, \zeta_{1}, \ldots, \zeta_{k}\right)$ nodes of $G^{\prime}$. Let a pair $\left(v^{\prime}, w^{\prime}\right)$ be an arc in $G^{\prime}$ if

- for every $i$, the head of the $\operatorname{arc} a_{i}$ is the tail of the $\operatorname{arc} b_{i}$;

- the paths $\left(a_{i}, b_{i}\right)$ for $i=1, \ldots, d$ are node disjoint; 
- for every $j=1, \ldots, k, \zeta_{j}=1$ if and only if $\varepsilon_{j}=1$ or if the head of the arc $b_{j}$ is an allowed maintenance node for urgent unit $j$.

We define the cost of the $\operatorname{arc}\left(v^{\prime}, w^{\prime}\right)$ as $\sum_{i=1}^{d} c\left(b_{i}\right)$.

Then a system of $d$ disjoint paths in $G$ covering all the red nodes and satisfying all the maintenance requirements corresponds to an $s^{\prime}-T^{\prime}$-path in $G^{\prime}$ with the same cost value, and vice versa.

\subsection{MR-graphs with one urgent unit}

We have seen in Section 4.1 that the feasibility problem with a single urgent node is NP-complete in artificially constructed graphs. It is, however, easy to solve in MR-graphs.

Theorem 3. Let $G=(V, A)$ be an MR-graph and let $A_{0}$ be the set of covered arcs. Moreover, let $s, t \in V$ and suppose that $P$ is an $s-t$-path in $G_{0}=\left(V, A_{0}\right)$ with inclusionwise minimal node set. Then there exists a path cover containing $P$.

Proof. Let $R$ denote the set of regular transitions. Consider the auxiliary graph $\widehat{G}$ of $G$ as defined in Section 3.2. Let $W$ be the set of nodes $v_{\text {out }}$ with $v$ in $P$ and $v \neq t$, and let $W_{N}$ be the set of nodes $v_{\text {out }} \in W$ with $v w \in P \backslash R$.

We show that no pair of different nodes $v_{\text {out }} \in W_{N}$ and $v_{\text {out }}^{\prime} \in W$ lie in the same strong component of $\widehat{G}$.

Suppose the contrary. Let $v w$ and $v^{\prime} w^{\prime}$ be arcs in path $P$. We may assume that $v^{\prime}$ lies on $P$ between $v$ and $t$, the other case being analogous. Let $K$ be the strong component of $\widehat{G}$ that contains $v_{\text {out }}$ and $v_{\text {out }}^{\prime}$. Then $w_{\text {in }} \in K$ since $v_{\text {out }} w_{\text {in }}$ belongs to a directed circuit in $\widehat{G}$. Moreover, if $v^{\prime} w^{\prime}$ is a regular transition, then every $v_{\text {out }}-v_{\text {out }}^{\prime}$-path contains the node $w_{\text {in }}^{\prime}$ since $w_{\text {in }}^{\prime} v_{\text {out }}^{\prime}$ is the only arc that enters $v_{\text {out }}^{\prime}$. If, however, $v^{\prime} w^{\prime}$ is a candidate transition, then $v_{\text {out }}^{\prime} w_{\text {in }}^{\prime}$ is contained in a circuit of $\widehat{G}$. In both cases we have $w_{\text {in }}^{\prime} \in K$. In particular, $\widehat{G}$ contains a $w_{\text {in }}^{\prime}-v_{\text {out }}$-path $\widehat{P}$.

For every $\operatorname{arc} x_{\text {out }} y_{\text {in }} \in \widehat{A}$, we have $\operatorname{Arr} \_s t a t i o n(x)=\operatorname{Dep} \_s t a t i o n(y)$, and for every $\operatorname{arc} x_{\text {in }} y_{\text {out }} \in \widehat{A}$, we have $\operatorname{Dep\_ station}(x)=\operatorname{Arr}$ _station $(y)$. Therefore $\operatorname{Arr} \_\operatorname{station}(v)=$ Dep_station $\left(w^{\prime}\right)$. Moreover,

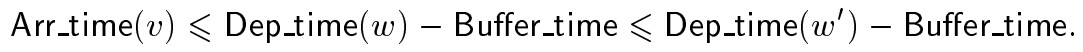

Thus $v w^{\prime}$ is either a regular transition (and thus it is covered) or a candidate transition. In the latter case, $\widehat{P} \cup\left\{v_{\text {out }} w_{\text {in }}^{\prime}\right\}$ is a circuit in $\widehat{G}$. Therefore $v w^{\prime}$ is 
covered. This contradicts the minimality of the path $P$. Therefore $v_{\text {out }}$ and $v_{\text {out }}^{\prime}$ cannot lie in the same strong component of $\widehat{G}$.

For every arc $v w \in P \backslash R$, fix a directed circuit $C_{v, w}$ in $\widehat{G}$ containing the arc $v_{\text {out }} w_{\text {in }}$. Let $C$ be the set of those edges in $G$ that correspond in $\widehat{G}$ to an edge in $\bigcup\left\{C_{v, w}: v w \in P \backslash R\right\}$. Then the circuits $C_{v, w}$ are node disjoint, therefore $(R \backslash C) \cup(C \backslash R)$ is a path cover of $G$. Moreover, no $\operatorname{arc}$ in $P \cap R$ belongs to $C$, therefore $(R \backslash C) \cup(C \backslash R)$ contains $P$.

This implies

Corollary 4. The feasibility problem of the Transition Model in an MR-graph with one urgent node (train unit) can be solved in polynomial time.

The NP-hardness of the feasibility problem in arbitrary acyclic graphs is partly caused by sparsity of acyclic graphs that are not MR-graphs. The proof above is based on the property of MR-graphs that the existence of a certain pair of $\operatorname{arcs} v w$ and $v^{\prime} w^{\prime}$ implies the existence of the arc $v w^{\prime}$.

The algorithm for the feasibility problem does not extend to the optimisation version of the single urgent unit case. Reason is that the cost of a path serving the urgent unit does not reflect the cost of a corresponding solution, as also non-urgent units will use new transitions, and these do contribute to the cost of the solution as well. Actually, finding an optimal solution to the Transition Model with one urgent node is also NP-hard in MR-graphs.

Theorem 5. It is NP-hard to find an optimal solution to the transition model on MR-graphs with one urgent train unit, one maintenance task and $\{0,1\}$ valued cost function.

Proof. First we show that each acyclic directed graph $G$ with a path cover $R$ can be extended to an MR-graph $G^{\prime}$. Indeed, there is only one station, and the arcs in $R$ are the regular transitions. We define departure and arrival times as follows. Consider a topological order of $G$, that is, an order on $V=\left\{v_{1}, \ldots, v_{n}\right\}$ such that $v_{i} v_{j} \in A$ implies $i<j$. Let Dep_time $\left(v_{i}\right)=2 i-1$, Arr_time $\left(v_{i}\right)=2 i$, and let Buffer_time $=0$. Then every arc $v w \in A$ is a regular or candidate transition. We insert an arc for all the other pairs $(v, w)$ that form a candidate transition according to the definition in Section 3.1. The obtained graph $G^{\prime}$ is an MR-graph.

We apply this to the graph $G$ constructed in the proof of Theorem 1 . We set the cost value 0 for the original arcs and we set the cost value 1 for the newly 
inserted arcs. Then deciding whether or not the optimisation problem in the extended graph $G^{\prime}$ has optimum value zero amounts to solving the feasibility problem in $G$.

\section{Computational results}

\subsection{The cost values}

The success of the model in practical applications highly depends on the choice of the cost function. We assume that the regular plan has a corresponding shunting plan that can be carried out in practice. The cost values measure how much the regular plan has to be modified in order to carry out the given transitions. We determined these transition costs from the timetable data, by taking the following factors into account:

- the positions of the units in the train compositions;

- the time difference between the tasks;

- information about the infrastructure like physical distance between the arrival and departure tracks;

- the station where the transition takes place; stations with heavy traffic provide less changing possibilities;

- the time that the transition is carried out; changes during rush hours are hard, while changes at night are typically easy.

\section{$5.2 \quad$ Experiments}

We used the train units of type 'Sprinter' as test example. NS Reizigers runs 47 train units of this type. The dimensions of the problem are given in Table 1. We can see that the reduction step described in Section 3 results in decreasing the number of arcs dramatically. Without this reduction, the binary linear programs could not be used at all. There are two maintenance tasks on every day. For simplicity, we set Buffer_time uniformly to 10 minutes, based on discussions with the planners.

We considered planning horizons of 2 up to 5 days. We generated 1000 instances per planning horizon. We selected urgent train units randomly: each with a fixed maintenance day, 2 urgent units per day in the planning period. 


\begin{tabular}{l|r|r|r|r}
\hline Planning period & 2 days & 3 days & 4 days & 5 days \\
\hline \# of tasks & 1,196 & 1,762 & 2,326 & 2,709 \\
\# of transitions & 98,035 & 198,614 & 332,031 & 508,853 \\
\# of covered transitions & 11,394 & 17,067 & 22,830 & 28,570 \\
\hline
\end{tabular}

Table 1

\begin{tabular}{l|c|c|c|c}
\hline Planning period & 2 days & 3 days & 4 days & 5 days \\
\hline \# urgent units & 4 & 6 & 8 & 10 \\
\# variables in IP & 28,453 & 51,193 & 79,786 & 114,096 \\
\# constraints in IP & 15,337 & 23,816 & 32,947 & 42,672 \\
Average solving time & $4.31 \mathrm{~s}$ & $8.92 \mathrm{~s}$ & $19.69 \mathrm{~s}$ & $43.03 \mathrm{~s}$ \\
Max. solving time & $6 \mathrm{~s}$ & $12 \mathrm{~s}$ & $138 \mathrm{~s}$ & $486 \mathrm{~s}$ \\
\hline
\end{tabular}

Table 2

Since the maintenance tasks start at the same time at the same location on every day, the integer program requires only one flow $x_{u}$ for every possible deadline.

For our computations we used the modelling software ILOG OPL Studio 3.7 and the integer program solver ILOG CPLEX 9.0 on a PC with an Intel P4 3.0 $\mathrm{GHz}$ processor and $512 \mathrm{MB}$ internal memory. Our results are summarised in Table 2.

Despite the large sizes of the integer linear programs, optimal solutions could be found in reasonably short time. It indicates that, although one can create difficult instances, the network structure of the problem allows a tight linear programming description for our test instances. Then, after a small number of branchings, CPLEX was able to construct an optimal integer solution.

In order to compare the Transition Model to the more involved Scenario Model that is described in [11], we derived the transition costs from the Scenario Model and modified slightly the objective function. Then the Transition Model becomes a relaxation of the Scenario Model. When solving the same instances in both models, the difference between the objective values turned out to be small: in all cases less than the cost of just one considerably expensive arc. That is, the solutions to both models hardly differ, although the Transition Model takes much less explicit details about shunting into account.

The practical value of the solutions, however, cannot be evaluated easily. A small number of examples has been discussed with the maintenance planners. They found the solutions satisfactory, although this does not mean that the 
local shunting crew would accept all these solutions. In this respect, the solutions generated by the model are comparable to the solutions generated by the maintenance planners themselves.

\section{Summary}

In this paper we described the Transition Model for routing passenger train units towards a maintenance facility. We analysed the computational complexity of this maintenance routing problem. In our computational tests, optimal solutions could be found in reasonably short running times by the commercial MIP solver CPLEX.

The Transition Model takes shunting into account via the arc cost values, resulting in an intuitive model which is much less involved than the Scenario Model described by Maróti and Kroon [11]. An advantage of the Transition Model is the fact that the required data can be collected more easily than in the case of the Scenario Model. A disadvantage may be that the probability of obtaining solutions that are feasible in the model but infeasible in practice is somewhat higher. Our computational experience showed that the solutions obtained from the two models do not differ much. Based on discussions with the maintenance planners in practice, we believe that the Transition Model is an appropriate basis for a decision support system for maintenance planning.

\section{Acknowledgement}

This research was supported by the Human Potential Programme of the European Union under contract no. HPRN-CT-1999-00104 (AMORE).

We would like to thank Bert Gerards and Lex Schrijver for their valuable comments on this paper.

\section{References}

[1] L. Anderegg, S. Eidenbenz, M. Gantenbein, Ch. Stamm, D.S. Taylor, B. Weber, P. Widmayer, Train routing algorithms: Concepts, design choices, and practical considerations, Proceedings of ALENEX'03, www. siam.org/meetings/alenex03/Abstracts/LAnderegg3.pdf. 
[2] C. Barnhart, N.L. Boland, L.W. Clarke, E. Johnson, G.L. Nemhauser, R.G. Shenoi, Flight string models for aircraft fleeting and routing, Transportation Science 32 No. 3 (1998), pp. 208-220.

[3] A.A. Bertossi, P. Carraresi, G. Gallo, On some matching problems arising in vehicle scheduling models, Networks 17 (1987), pp. 271-281.

[4] P. Carraresi, G. Gallo, Network models for vehicle and crew scheduling, European Journal of Operational Research 16 (1984), pp. 139-151.

[5] L. Clarke, E. Johnson, G. Nemhauser, Z. Zhu, The aircraft rotation problem, Annals of Operations Research 69 (1997), pp. 33-46.

[6] S.A. Cook, The complexity of theorem-proving procedures, in: Conference Record of Third Annual ACM Symposium on Theory of Computing The Association for Computing Machinery, New York, 1971, pp 151-158.

[7] J. Desrosiers, Y. Dumas, M.M. Solomon, F. Soumis, Time constrained routing and scheduling, In: Handbooks in OR and MS, vol. 8, pp. 35-139, Elsevier, 1995.

[8] T.A. Feo, J.F. Bard, Flight scheduling and maintenance base planning, Management Science 35 No. 12 (1989), pp. 1514-1532.

[9] R. Gopalan, K.T. Talluri, The aircraft maintenance routing problem, $O p$ erations Research 46 No. 2 (1998), pp. 260-271.

[10] N. Lingaya, J.F. Cordeau, G. Desaulniers, J. Desrosiers, F. Soumis, Operational car assignment at VIA Rail Canada, Transportation Research Part B 36 (2002) pp. $755-778$.

[11] G. Maróti, L.G. Kroon, Maintenance routing for train units: the Scenario Model, CWI Research Report, 2004

[12] A. Schrijver, Combinatorial Optimization: Polyhedra and efficiency, Springer Verlag, Berlin, Heidelberg, 2003.

[13] K.T. Talluri, The four-day aircraft maintenance routing problem, Transportation Science 32 No. 1 (1998), pp. 43-53.

[14] P. Toth, D. Vigo, The vehicle routing problem, SIAM Monographs on Discrete Mathematics and Applications, 2002. 